\title{
ONE-SIDED $L$-APPROXIMATION ON A SPHERE OF THE CHARACTERISTIC FUNCTION OF A LAYER
}

\author{
Marina V. Deikalova ${ }^{\dagger}$ and Anastasiya Yu. Torgashova ${ }^{\dagger \dagger}$ \\ Ural Federal University, \\ 51 Lenin aven., Ekaterinburg, Russia, 620000 \\ ${ }^{\dagger}$ marina.deikalova@urfu.ru, ††anastasiya.torgashova@mail.ru
}

\begin{abstract}
In the space $L\left(\mathbb{S}^{m-1}\right)$ of functions integrable on the unit sphere $\mathbb{S}^{m-1}$ of the Euclidean space $\mathbb{R}^{m}$ of dimension $m \geq 3$, we discuss the problem of one-sided approximation to the characteristic function of a spherical layer $\mathbb{G}(J)=\left\{x=\left(x_{1}, x_{2}, \ldots, x_{m}\right) \in \mathbb{S}^{m-1}: x_{m} \in J\right\}$, where $J$ is one of the intervals $(a, 1],(a, b)$, and $[-1, b),-1<a<b<1$, by the set of algebraic polynomials of given degree $n$ in $m$ variables. This problem reduces to the one-dimensional problem of one-sided approximation in the space $L^{\phi}(-1,1)$ with the ultraspherical weight $\phi(t)=\left(1-t^{2}\right)^{\alpha}, \alpha=(m-3) / 2$, to the characteristic function of the interval $J$. This result gives a solution of the problem of one-sided approximation to the characteristic function of a spherical layer in all cases when a solution of the corresponding one-dimensional problem known. In the present paper, we use results by A.G. Babenko, M.V. Deikalova, and Sz.G. Revesz (2015) and M.V. Deikalova and A.Yu. Torgashova (2018) on the one-sided approximation to the characteristic functions of intervals.
\end{abstract}

Keywords: One-sided approximation, characteristic function, spherical layer, spherical cap, algebraic polynomials.

\section{Introduction}

Let $\mathbb{R}^{m}, m \geq 2$, be the Euclidean space with the inner product

$$
x y=\sum_{i=1}^{m} x_{k} y_{k}, \quad x=\left(x_{1}, x_{2}, \ldots, x_{m}\right), \quad y=\left(y_{1}, y_{2}, \ldots, y_{m}\right),
$$

and the norm $\|x\|=\sqrt{x x}$. For $r>0$, we consider in the space $\mathbb{R}^{m}$ the sphere $\mathbb{S}^{m-1}(r)=\left\{x \in \mathbb{R}^{m}\right.$ : $\|x\|=r\}$ of radius $r$ centered at the origin; we denote by $\mathbb{S}^{m-1}$ the unit sphere $(r=1)$. For $-1 \leq a<b \leq 1$, we define the intervals

$$
J= \begin{cases}(a, b), & -1<a<b<1 ; \\ (a, 1], & -1<a<b=1 ; \\ {[-1, b),} & a=-1<b<1 .\end{cases}
$$

By means of these intervals, we define the spherical layers

$$
\mathbb{G}(J)=\left\{x=\left(x_{1}, x_{2}, \ldots, x_{m}\right) \in \mathbb{S}^{m-1}: x_{m} \in J\right\}
$$

centered at the "north pole" $e_{m}=(0,0, \ldots, 0,1)$ of the sphere. In the case $b=1$ and $-1<a<1$, the layer

$$
\mathbb{C}(a)=\mathbb{G}(a, 1]=\left\{x=\left(x_{1}, x_{2}, \ldots, x_{m}\right) \in \mathbb{S}^{m-1}: x_{m} \in(a, 1]\right\}
$$

\footnotetext{
${ }^{1}$ This work was supported by the Russian Foundation for Basic Research (project no. 18-01-00336) and by the Russian Academic Excellence Project (agreement no. 02.A03.21.0006 of August 27, 2013, between the Ministry of Education and Science of the Russian Federation and Ural Federal University).
} 
is the spherical cap.

Let $\mathbb{H}$ be one of the following manifolds: either an interval from the real line or a sphere $\mathbb{S}^{k-1}(r)$ of radius $r>0$ in the space $\mathbb{R}^{k}, 2 \leq k \leq m$, in particular, the unit sphere $\mathbb{S}^{m-1}=\mathbb{S}^{m-1}(1)$. On each of these sets, we consider the classical Lebesgue measure (of corresponding dimension). For a measurable subset $E \subset \mathbb{H}$, denote by $|E|$ the (corresponding) measure of the set $E$. Let $L(E)$ be the space of functions measurable and integrable on $E$. For a function $f \in L(E)$, its Lebesgue integral on the set $E$ is written as $\int_{E} f(x) d x$. We assume that the space $L(E)$ is equipped with the norm $\|f\|=\|f\|_{L(E)}=\int_{E}|f(x)| d x$.

Denote by $\mathscr{P}_{n, m}$ the set of algebraic polynomials

$$
\begin{gathered}
P_{n}(x)=\sum_{\substack{\alpha \mid=\alpha_{1}+\cdots+\alpha_{m} \leq n, \alpha=\left(\alpha_{1}, \ldots, \alpha_{m}\right) \in \mathbb{Z}_{+}^{m}}} c_{\alpha} x^{\alpha}, \\
x^{\alpha}=x_{1}^{\alpha_{1}} x_{2}^{\alpha_{2}} \cdots x_{m}^{\alpha_{m}}, \quad x=\left(x_{1}, x_{2}, \ldots, x_{m}\right) \in \mathbb{R}^{m},
\end{gathered}
$$

of degree (at most) $n$ in $m$ variables with real coefficients $c_{\alpha}$.

In what follows, for a couple of measurable functions $f$ and $g$ on the sphere $\mathbb{S}^{m-1}$, the inequality $f \leq g$ means that $f(x) \leq g(x)$ for almost all $x \in \mathbb{S}^{m-1}$. Given a function $f$ defined and measurable on the sphere $\mathbb{S}^{m-1}$, we consider the sets

$$
\mathscr{P}_{n, m}^{-}(f)=\left\{P_{n} \in \mathscr{P}_{n, m}: P_{n} \leq f\right\}, \quad \mathscr{P}_{n, m}^{+}(f)=\left\{P_{n} \in \mathscr{P}_{n, m}: P_{n} \geq f\right\}
$$

of polynomials from $\mathscr{P}_{n, m}$ whose graphs "lie" under and over the graph of the function $f$, respectively. In order to the sets (0.3) were not empty, we assume that $f$ is lower bounded in the former case and upper bounded in the latter case. Consider the values of the best one-sided approximation in the space $L\left(\mathbb{S}^{m-1}\right)$ to the function $f$ from below and from above by the set $\mathscr{P}_{n, m}$ :

$$
e_{n, m}^{\mp}(f)=\inf \left\{\left\|f-P_{n}\right\|_{L\left(\mathbb{S}^{m-1}\right)}: P_{n} \in \mathscr{P}_{n, m}^{\mp}(f)\right\} .
$$

Polynomials implementing the infimums in these relations are called polynomials of the best (integral) approximation to the function $f$ from below and from above, respectively, or simply extremal polynomials. The space $\mathscr{P}_{n, m}$ is finite-dimensional; therefore, as easily understood, extremal polynomials in (0.4) exist; i. e., we can replace inf by min in (0.4) (as well as in similar problems in what follows).

The main aim of the present paper is to study the best one-sided approximation from below in the space $L\left(\mathbb{S}^{m-1}\right)$ to the characteristic function

$$
\mathbf{1}_{\mathbb{G}(J)}(x)= \begin{cases}1, & x \in \mathbb{G}(J), \\ 0, & x \notin \mathbb{G}(J),\end{cases}
$$

of a spherical layer $(0.2)$ by the subspace of polynomials $\mathscr{P}_{n, m}$; more exactly, to study the value

$$
e_{n, m}^{-}\left(\mathbf{1}_{\mathbb{G}(J)}\right)=\inf \left\{\left\|\mathbf{1}_{\mathbb{G}(J)}-P_{n}\right\|_{L\left(\mathbb{S}^{m-1}\right)}: P_{n} \in \mathscr{P}_{n, m}^{-}\left(\mathbf{1}_{\mathbb{G}(J)}\right)\right\} ;
$$

here, in accordance with the introduced notation,

$$
\mathscr{P}_{n, m}^{-}\left(\mathbf{1}_{\mathbb{G}(J)}\right)=\left\{P_{n} \in \mathscr{P}_{n, m}: P_{n} \leq \mathbf{1}_{\mathbb{G}(J)}\right\} .
$$

The fact that function (0.5) is zonal plays an important role in what follows. In this paper, a function $f(x), x=\left(x_{1}, x_{2}, \ldots, x_{m}\right)$, defined on the sphere $\mathbb{S}^{m-1}$ is called zonal if it depends only on the coordinate $x_{m}$ of the point $x=\left(x_{1}, x_{2}, \ldots, x_{m}\right) \in \mathbb{S}^{m-1}$, i. e.,

$$
f\left(x_{1}, x_{2}, \ldots, x_{m}\right)=\zeta\left(x_{m}\right), \quad x=\left(x_{1}, x_{2}, \ldots, x_{m}\right) \in \mathbb{S}^{m-1},
$$


where $\zeta$ is a univariate function defined on the interval $[-1,1]$. For function $(0.5)$, the function $\zeta$ in representation (0.7) is the characteristic function of the interval (0.1).

In the present paper, we only discuss problems of one-sided approximation from below. The problems of approximation from above are not specially considered. However, in certain cases, results for approximations from above can be obtained either as consequences of results on the approximation from below or by the same scheme.

Approximation without constraints in the space $L\left(\mathbb{S}^{m-1}\right)$ to the characteristic function of a spherical layer and a spherical cap by algebraic polynomials was studied by one of the authors [8-10]. Note also that, by now, there are many studies devoted to approximation theory, harmonic analysis, and extremal problems on Euclidean sphere; see, for example, monographs [7, 13] and the references therein.

\section{Reduction to a one-dimensional problem}

In this section, we show that, by means of averaging, problems (0.4) of one-sided approximation to a zonal function in the space $L\left(\mathbb{S}^{m-1}\right)$ on the sphere reduce to problems of one-sided approximation to the corresponding univariate function in the space $L^{\phi}(-1,1)$ of functions integrable on the interval $(-1,1)$ with the ultraspherical weight

$$
\phi(t)=\left(1-t^{2}\right)^{\alpha}, \quad \alpha=\frac{m-3}{2},
$$

which is equipped with the norm

$$
\|g\|_{L^{\phi}(-1,1)}=\int_{-1}^{1}|g(t)| \phi(t) d t
$$

To prove this fact, we apply an averaging operator $\Upsilon$, which, to a function $f \in L\left(\mathbb{S}^{m-1}\right)$, set in correspondence a function of one variable $t \in(-1,1)$ by the formula

$$
(\Upsilon f)(t)=\frac{1}{\left|\mathbb{S}^{m-2}\right|} \int_{\mathbb{S}^{m-2}} f\left(\sqrt{1-t^{2}} \widetilde{x}, t\right) d \widetilde{x}
$$

The following lemma implies that this is a bounded linear operator from the space $L\left(\mathbb{S}^{m-1}\right)$ to the space $L^{\phi}(-1,1)$. This lemma is a variant of Fubini's theorem (see, for example, [12, Ch. III, Sect. 11]); a detailed proof of this statement with the passage to polar coordinates on the sphere can be found, for example, in [8].

Lemma 1. The following statements hold for a function $f$ integrable on the sphere $\mathbb{S}^{m-1}$ for $m \geq 3$.

(1) For almost all $t \in(-1,1)$, the function $f\left(\sqrt{1-t^{2}} \widetilde{x}, t\right)$ of variable $\widetilde{x} \in \mathbb{S}^{m-2}$ is integrable on $\mathbb{S}^{m-2}$.

(2) The function

$$
g(t)=\frac{1}{\left|\mathbb{S}^{m-2}\right|} \int_{\mathbb{S}^{m-2}} f\left(\sqrt{1-t^{2}} \widetilde{x}, t\right) d \widetilde{x}
$$

of variable $t \in(-1,1)$ is integrable with ultraspherical weight $(1.1)$ on the interval $(-1,1)$; i.e., $g \in L^{\phi}(-1,1)$.

(3) The following formula holds:

$$
\int_{\mathbb{S}^{m-1}} f(x) d x=\left|\mathbb{S}^{m-2}\right| \int_{-1}^{1} g(t)\left(1-t^{2}\right)^{(m-3) / 2} d t .
$$


According to Lemma 1, the averaging operator $\Upsilon$ is well defined by formula (1.2) on the whole space $L\left(\mathbb{S}^{m-1}\right)$ and is a linear operator from $L\left(\mathbb{S}^{m-1}\right)$ to $L^{\phi}(-1,1)$. The following estimate holds for $f \in L\left(\mathbb{S}^{m-1}\right)$ for almost all points $t \in(-1,1)$ :

$$
|g(t)|=|(\Upsilon f)(t)| \leq \widetilde{g}(t)=\frac{1}{\left|\mathbb{S}^{m-2}\right|} \int_{\mathbb{S}^{m-2}}\left|f\left(\sqrt{1-t^{2}} \widetilde{x}, t\right)\right| d \widetilde{x}=(\Upsilon|f|)(t) .
$$

Hence, using formula (1.4) for the function $|f|$, we obtain the inequality

$$
\left|\mathbb{S}^{m-2}\right|\|g\|_{L^{\phi}(-1,1)}=\left|\mathbb{S}^{m-2}\right| \int_{-1}^{1}|g(t)|\left(1-t^{2}\right)^{(m-3) / 2} d t \leq\|f\|_{L\left(\mathbb{S}^{m-1}\right)} .
$$

The latter inequality turns into an equality at least in the following two cases: (1) the function $f$ is nonnegative; (2) the function $f$ is zonal, more exactly, depends only on the variable $x_{m}$. Indeed, if $f$ is nonnegative, then the unique inequality in the chain of relations given above turns into an equality. In the case when the function $f$ is zonal, we have $\Upsilon f=f$, i. e., $f(x)=g\left(x_{m}\right)$ and, obviously, inequality (1.5) turns into an equality.

Thus, the averaging operator $(1.2)$ is a bounded linear operator from $L\left(\mathbb{S}^{m-1}\right)$ to $L^{\phi}(-1,1)$ and the equality $\left|\mathbb{S}^{m-2}\right|\|\Upsilon\|=1$ holds for its norm $\|\Upsilon\|$.

Note also that, if the function $f$ is continuous on the sphere $\mathbb{S}^{m-1}$, then the function $g=\Upsilon f$ is continuous on the interval $\mathbb{I}=[-1,1]$ and $g(1)=f\left(e_{m}\right), e_{m}=(0,0, \ldots, 0,1)$.

The following lemma describes the structure of function (1.3) in the case when $f$ is a polynomial. The proof of the lemma can also be found in [8].

Lemma 2. For $n \geq 1$ and $m \geq 3$, for any polynomial $P_{n} \in \mathscr{P}_{n, m}$, the function

$$
g_{n}(t)=\left(\Upsilon P_{n}\right)(t)=\frac{1}{\left|\mathbb{S}^{m-2}\right|} \int_{\mathbb{S}^{m-2}} P_{n}\left(\sqrt{1-t^{2}} \widetilde{x}, t\right) d \widetilde{x}
$$

is a polynomial in (one) variable $t=x_{m}$ of degree at most $n$.

Thus, we have the embedding $\Upsilon \mathscr{P}_{n, m} \subset \mathscr{P}_{n}$, where $\mathscr{P}_{n}=\mathscr{P}_{n, 1}$ is the set of polynomials in one variable of degree at most $n$. In fact, we have the equality

$$
\Upsilon \mathscr{P}_{n, m}=\mathscr{P}_{n} .
$$

Indeed, a polynomial $p_{n} \in \mathscr{P}_{n}$ can be regarded as a polynomial in $m$ variables; more exactly, with the polynomial $p_{n}$, we associate the polynomial $P_{n}\left(x_{1}, x_{2}, \ldots, x_{m}\right)=p_{n}\left(x_{m}\right)$ in $m$ variables. In this case, the right-hand side of (1.6) is the polynomial $p_{n}$; thus, $\Upsilon P_{n}=p_{n}$. Relation (1.7) is verified.

Lemma 3. Let $m \geq 3$. If a function $f$ is defined, integrable, and lower bounded on the sphere $\mathbb{S}^{m-1}$, then the embedding $\Upsilon\left(\mathscr{P}_{n, m}^{-}(f)\right) \subset \mathscr{P}_{n}^{-}(\Upsilon f)$ holds. If, in addition, the function $f$ is zonal, then the following equality holds:

$$
\Upsilon\left(\mathscr{P}_{n, m}^{-}(f)\right)=\mathscr{P}_{n}^{-}(\Upsilon f) .
$$

P r o o f. For the beginning, let $f$ be an arbitrary integrable and lower bounded (not necessarily zonal) function on the sphere, and let $P_{n} \in \mathscr{P}_{n, m}^{-}(f)$. Recall that the property $P_{n} \in \mathscr{P}_{n, m}^{-}(f)$ means that the set $\Omega=\Omega\left(f, P_{n}\right)$ of points $x \in \mathbb{S}^{m-1}$ at which the inequality $P_{n}(x) \leq f(x)$ holds is of full measure (i. e., the difference $\mathbb{S}^{m-1} \backslash \Omega$ is a null-measure set) in $\mathbb{S}^{m-1}$.

We have to compare the averaging of the polynomial $P_{n}$

$$
\left(\Upsilon P_{n}\right)(t)=\frac{1}{\left|\mathbb{S}^{m-2}\right|} \int_{\mathbb{S}^{m-2}} P_{n}\left(\sqrt{1-t^{2}} \widetilde{x}, t\right) d \widetilde{x}
$$


and the averaging of the function $f$

$$
(\Upsilon f)(t)=\frac{1}{\left|\mathbb{S}^{m-2}\right|} \int_{\mathbb{S}^{m-2}} f\left(\sqrt{1-t^{2}} \widetilde{x}, t\right) d \widetilde{x}
$$

for (almost all) $t \in(-1,1)$.

Since a polynomial $P_{n}$ is a continuous function, averaging (1.8) exists for all points $t \in[-1,1]$; moreover, according to Lemma $2,\left(\Upsilon P_{n}\right)(t)$ is a polynomial in one variable. Averaging (1.9) exists, in general, not for all $t \in(-1,1)$. According to Lemma 1, integrals (1.9) exist for almost all $t \in(-1,1)$, i. e., everywhere on $(-1,1)$ except for a null-measure set $I_{0}^{\prime} \subset(-1,1)$.

Let us study the set of pairs $(\widetilde{x}, t)$ of points at which the following inequality holds:

$$
P_{n}\left(\sqrt{1-t^{2}} \widetilde{x}, t\right) \leq f\left(\sqrt{1-t^{2}} \widetilde{x}, t\right) .
$$

Let us ascertain that this inequality holds for almost all $t \in(-1,1)$ (i. e., everywhere on $(-1,1)$ except for a null-measure set $I_{0}^{\prime \prime}$ ) for almost all $\widetilde{x} \in \mathbb{S}^{m-2}$ (i. e., everywhere on $\mathbb{S}^{m-2}$ except for a null-measure set $\left.S_{0}(t)\right)$. In other words, the set

$$
\omega(t)=\left\{\widetilde{x} \in \mathbb{S}^{m-2}:\left(\sqrt{1-t^{2}} \widetilde{x}, t\right) \in \Omega\right\}
$$

is of full measure in $\mathbb{S}^{m-2}$ for almost all $t \in(-1,1)$. This fact can be proved, for example, on the following way.

Let us apply Lemma 1 to the characteristic function $\mathbf{1}_{\Omega}$ of the set $\Omega$. By the first statement of the lemma, the set $\omega(t)$ is measurable on $\mathbb{S}^{m-2}$ for almost all $t \in(-1,1)$ (i. e., everywhere on $(-1,1)$ except for a null-measure set $\left.I_{0}^{\prime \prime}\right)$. Formula (1.4) for the function $\mathbf{1}_{\Omega}$ takes the form

$$
\left|\mathbb{S}^{m-1}\right|=\int_{-1}^{1}|\omega(t)|_{m-2}\left(1-t^{2}\right)^{(m-3) / 2} d t .
$$

For the measure of set (1.10), we have the estimate

$$
|\omega(t)|_{m-2} \leq\left|\mathbb{S}^{m-2}\right|
$$

Therefore, (1.11) implies that

$$
\left|\mathbb{S}^{m-1}\right|=\int_{-1}^{1}|\omega(t)|_{m-2}\left(1-t^{2}\right)^{(m-3) / 2} d t \leq\left|\mathbb{S}^{m-2}\right| \int_{-1}^{1}\left(1-t^{2}\right)^{(m-3) / 2} d t .
$$

The latter value is $\left|\mathbb{S}^{m-1}\right|$. Hence, inequality (1.12) turns into an equality on the set $(-1,1) \backslash I_{0}^{\prime \prime}$. Thus, set $(1.10)$ is measurable for almost all $t \in(-1,1)$ and

$$
|\omega(t)|_{m-2}=\left|\mathbb{S}^{m-2}\right| \text {. }
$$

The set $I_{0}=I_{0}^{\prime} \bigcup I_{0}^{\prime \prime} \subset(-1,1)$ is a null-measure set; (1.9) holds for points $t \notin I_{0}$, the set (1.10) is measurable, and (1.13) hods. For points $t \in(-1,1) \backslash I_{0}$, the inequality $\left(\Upsilon P_{n}\right)(t) \leq(\Upsilon f)(t)$ holds.

Consequently, the embedding $\Upsilon\left(\mathscr{P}_{n, m}^{-}(f)\right) \subset \mathscr{P}_{n}^{-}(\Upsilon f)$ holds. Most likely, the inverse embedding does not hold in the general case.

Assume that a function $f$ is zonal and, moreover, can be represented in the form (0.7). A polynomial $p_{n} \in \mathscr{P}_{n}^{-}(g)$ regarded as a zonal polynomial, obviously, belongs to the set $\mathscr{P}_{n, m}^{-}(f)$ and $\Upsilon p_{n}=p_{n}$. Therefore, in this case, the inverse embedding $\mathscr{P}_{n}^{-}(g) \subset \Upsilon\left(\mathscr{P}_{n, m}^{-}(f)\right)$ hods. Lemma 3 is proved.

For a function $g \in L^{\phi}(-1,1)$ lower bounded on $(-1,1)$, consider the value

$$
E_{n, \phi}^{-}(g)=\inf \left\{\|g-p\|_{L^{\phi}(-1,1)}: p \in \mathscr{P}_{n}^{-}(f)\right\}
$$

of its best approximation from below in the space $L^{\phi}(-1,1)$ by the set $\mathscr{P}_{n}$. 
Theorem 1. Let $m \geq 3$ and $n \geq 0$. For an arbitrary function $f \in L\left(\mathbb{S}^{m-1}\right)$ lower bounded on $\mathbb{S}^{m-1}$, the following inequality holds:

$$
e_{n, m}^{-}(f) \geq\left|\mathbb{S}^{m-2}\right| E_{n, \phi}^{-}(\Upsilon f) .
$$

For a zonal function $f \in L\left(\mathbb{S}^{m-1}\right)$, the following equality holds:

$$
e_{n, m}^{-}(f)=\left|\mathbb{S}^{m-2}\right| E_{n, \phi}^{-}(\Upsilon f)
$$

and, if a polynomial $p_{n}^{*}$ in one variable is extremal in problem (1.14) for the function $g=\Upsilon f$, (i.e., the infimum in (1.14) is attained at this polynomial), then the zonal polynomial $P_{n}^{*}(x)=p_{n}^{*}\left(x_{m}\right)$, $x=\left(x_{1}, x_{2}, \ldots, x_{m}\right) \in \mathbb{R}^{m}$, is extremal in problem $(0.6)$.

P r o o f. Let $P_{n} \in \mathscr{P}_{n, m}^{-}(f)$, and let $g_{n}$ be the function (of one variable) constructed by formula (1.3):

$$
g_{n}(t)=\frac{1}{\left|\mathbb{S}^{m-2}\right|} \int_{\mathbb{S}^{m-2}} P_{n}\left(\sqrt{1-t^{2}} \widetilde{x}, t\right) d \widetilde{x} .
$$

As shown above, $g_{n} \in \mathscr{P}_{n}^{-}(g)$, where $g=\Upsilon f$. We have

$$
g(t)-g_{n}(t)=\frac{1}{\left|\mathbb{S}^{m-2}\right|} \int_{\mathbb{S}^{m-2}}\left(g(t)-P_{n}\left(\sqrt{1-t^{2}} \widetilde{x}, t\right)\right) d \widetilde{x}
$$

Hence,

$$
\left|\mathbb{S}^{m-2}\right| \times\left\|g-g_{n}\right\|_{L^{\phi}(-1,1)}=\left\|f-P_{n}\right\|_{L\left(\mathbb{S}^{m-1}\right)} .
$$

Indeed, the following chain of relations holds:

$$
\begin{gathered}
\left\|g-g_{n}\right\|_{L^{\phi}(-1,1)}=\int_{-1}^{1}\left|g(t)-g_{n}(t)\right|\left(1-t^{2}\right)^{(m-3) / 2} d t= \\
=\int_{-1}^{1}\left(g(t)-g_{n}(t)\right)\left(1-t^{2}\right)^{(m-3) / 2} d t= \\
=\int_{-1}^{1}\left(1-t^{2}\right)^{(m-3) / 2} \frac{1}{\left|\mathbb{S}^{m-2}\right|} \int_{\mathbb{S}^{m-2}}\left(f\left(\sqrt{1-t^{2}} \widetilde{x}, t\right)-P_{n}\left(\sqrt{1-t^{2}} \widetilde{x}, t\right)\right) d \widetilde{x} d t= \\
=\frac{1}{\left|\mathbb{S}^{m-2}\right|} \int_{\mathbb{S}^{m-1}}\left(f(x)-P_{n}(x)\right) d x=\frac{1}{\left|\mathbb{S}^{m-2}\right|} \int_{\mathbb{S}^{m-1}}\left|f(x)-P_{n}(x)\right| d x= \\
=\frac{1}{\left|\mathbb{S}^{m-2}\right|}\left\|f-P_{n}\right\|_{L\left(\mathbb{S}^{m-1}\right)} .
\end{gathered}
$$

This, by Lemma 3, implies relations (1.15) and (1.16). Due to equality (1.16), at the polynomial $P_{n}^{*}(x)=p_{n}^{*}\left(x_{m}\right), x=\left(x_{1}, x_{2}, \ldots, x_{m}\right) \in \mathbb{R}^{m}$, the infimum in (0.6) is attained; thus, this polynomial is extremal. Theorem 1 is proved.

Function (0.5) is zonal; more exactly,

$$
\mathbf{1}_{\mathbb{G}(J)}(x)=\mathbf{1}_{J}\left(x_{m}\right), \quad x=\left(x_{1}, x_{2}, \ldots, x_{m}\right) \in \mathbb{S}^{(m-1)},
$$

where $\mathbf{1}_{J}$ is the characteristic function of the interval (0.1):

$$
\mathbf{1}_{J}(t)= \begin{cases}1, & t \in J, \\ 0, & t \in[-1,1] \backslash J .\end{cases}
$$


Consider the best approximation from below

$$
E_{n, \phi}^{-}\left(\mathbf{1}_{J}\right)=\inf \left\{\left\|\mathbf{1}_{J}-p_{n}\right\|_{L^{\phi}(-1,1)}: p_{n} \in \mathscr{P}_{n}^{-}\left(\mathbf{1}_{J}\right)\right\}
$$

to the step function $\mathbf{1}_{J}$ in the space $L^{\phi}(-1,1)$ by the set $\mathscr{P}_{n}^{-}\left(\mathbf{1}_{J}\right)=\mathscr{P}_{n, 1}^{-}\left(\mathbf{1}_{J}\right)$ of algebraic polynomials (in one variable) whose graphs lie under the graph of the function $\mathbf{1}_{J}$. As a particular case of Theorem 1, the following statement is valid.

Theorem 2. For any $m \geq 3$ and $n \geq 0$, the following formula holds for intervals (0.1):

$$
e_{n, m}^{-}\left(\mathbf{1}_{\mathbb{G}(J)}\right)=\left|\mathbb{S}^{m-2}\right| E_{n, \phi}^{-}\left(\mathbf{1}_{J}\right)
$$

and, if a polynomial $p_{n}^{*}$ in one variable is extremal in problem (1.17), then the zonal polynomial $P_{n}^{*}(x)=p_{n}^{*}\left(x_{m}\right), x=\left(x_{1}, x_{2}, \ldots, x_{m}\right) \in \mathbb{R}^{m}$, is extremal in problem $(0.6)$.

\section{One-sided approximation to the characteristic function of an interval}

Let $v$ be a measurable integrable nonnegative function different from zero almost everywhere on $(-1,1)$; we will call such a function a weight (on $(-1,1)$ ). Denote by $L^{v}(-1,1)$ the space of real-valued functions $f$ integrable with weight $v$ on $(-1,1)$ equipped with the norm

$$
\|f\|=\|f\|_{L^{v}(-1,1)}=\int_{-1}^{1}|f(t)| v(t) d t .
$$

In this section, we give results from $[1,5,11]$ on the one-sided approximation in the space $L^{v}(-1,1)$ to the characteristic function of an interval by algebraic polynomials. The results from Section 1 and from this sections make it possible to find the best one-sided approximation in the space $L\left(\mathbb{S}^{m-1}\right)$ to the characteristic function of a spherical layer (in particular, a spherical cap) by algebraic polynomials in certain situations.

For nonnegative integer $n$, we denote by $\mathscr{P}_{n}$ the set of algebraic polynomials $p(t)=\sum_{k=0}^{n} a_{k} t^{k}$ in one real variable of degree at most $n$ with real coefficients.

In contrast to Section 1, in this section, for a couple of measurable functions $f$ and $g$ on the interval $[-1,1]$, the inequality $f \leq g$ means that $f(t) \leq g(t)$ for all $t \in[-1,1]$. For a function $f$ defined, bounded, and measurable on the interval $[-1,1]$, we consider the sets

$$
\mathscr{P}_{n}^{-}(f)=\left\{p \in \mathscr{P}_{n}: p \leq f\right\}, \quad \mathscr{P}_{n}^{+}(f)=\left\{p \in \mathscr{P}_{n}: p \geq f\right\}
$$

of polynomials from $\mathscr{P}_{n}$ whose graphs lie under and over over the graph of the function $f$, respectively. The function $f$ is assumed lower bounded in the former case and upper bounded in the latter case. We are interested in the values

$$
E_{n, v}^{\mp}(f)=\inf \left\{\|f-p\|_{L^{v}(-1,1)}: p \in \mathscr{P}_{n}^{\mp}(f)\right\}
$$

of the best approximation in the space $L^{v}(-1,1)$ to the function $f$ from below and from above by the set $\mathscr{P}_{n}$ as well as in extremal polynomials at which the infimums in (2.1) are attained.

An important tool for studying the one-sided approximation to functions by polynomials are quadrature formulas

$$
\int_{-1}^{1} v(t) p(t) d t=\sum_{k=1}^{M} \lambda_{k} p\left(t_{k}\right), \quad p \in \mathscr{P}_{n},
$$

exact on the set of polynomials $\mathscr{P}_{n}$ with nodes $-1 \leq t_{1}<t_{2}<\cdots<t_{M} \leq 1$ and positive weights: $\lambda_{k}>0,1 \leq k \leq M$; such quadrature formulas are called positive. The largest degree $n$ of 
polynomials for which formula (2.2) holds is called the degree of precision of this formula. Depending on a situation, some of nodes of formula (2.2) can be fixed while the remaining are assumed free; more exactly, they are chosen so that the formula have the highest degree of precision (see, for example, [15, Ch. 7, Sect. 1]. The following statement is due to Bojanic and DeVore [4, the proof of Theorem 2] (see also [14, Theorem 1.7.5]).

Theorem A. Assume that quadrature formula (2.2) holds on the set $\mathscr{P}_{n}$. Then, the following estimates are valid for a measurable bounded function $f$ :

$$
E_{n, v}^{-}(f) \geq \int_{-1}^{1} v(t) f(t) d t-\sum_{k=1}^{M} \lambda_{k} f\left(t_{k}\right), \quad E_{n, v}^{+}(f) \geq \sum_{k=1}^{M} \lambda_{k} f\left(t_{k}\right)-\int_{-1}^{1} v(t) f(t) d t .
$$

If an inequality in (2.3) turns into an equality, then quadrature formula (2.2) is said to be extremal in the corresponding problem (2.1).

Consider the problem of one-sided approximation to the characteristic function

$$
\mathbf{1}_{J}(t)= \begin{cases}1, & t \in J \\ 0, & t \in[-1,1] \backslash J\end{cases}
$$

of interval $(0.1)$ in the space $L^{v}(-1,1)$ by algebraic polynomials of given degree $n \geq 0$. The problem consists in finding the values

$$
E_{n}^{\mp}\left(\mathbf{1}_{J}\right)=E_{n, v}^{\mp}\left(\mathbf{1}_{J}\right)=\inf \left\{\left\|\mathbf{1}_{J}-p_{n}\right\|_{L^{v}(-1,1)}: p_{n} \in \mathscr{P}_{n}^{\mp}\left(\mathbf{1}_{J}\right)\right\} .
$$

Problems of one-sided weighted integral approximation to the characteristic function of an interval and to similar functions by algebraic or trigonometric polynomials arise in various areas of mathematics and have a rich history (see $[1,2,5,6,11,14,16,17]$ and the references therein). Let us outline only several exact results on problem (2.4) closely related to the present paper; for a more complete presentation of the topic see $[1,11]$. Problem $(2.4)$ of one-sided integral approximation to the characteristic function of an arbitrary half-open interval $(a, 1] \subset(-1,1]$ by algebraic polynomials on $[-1,1]$ with the unit weight was solved, and the whole class of extremal polynomials was described in [5]. This problem in the space $L^{v}(-1,1)$ with an arbitrary weight is solved in [1]. Let us describe the main result of [1] in the form convenient for us.

In the study of problems (2.4) of one-sided approximation to the characteristic function of an interval by polynomials, $M$-point quadrature formulas are used, the set $\mathfrak{u}$ of fixed nodes of which either contains no fixed nodes or contains one, two, or three fixed nodes of a specific form:

$$
\begin{gathered}
\varnothing, \quad\{-1\}, \quad\{1\}, \quad\{-1,1\}, \\
\{\theta\},\{-1, \theta\}, \quad\{\theta, 1\}, \quad\{-1, \theta, 1\}, \quad \text { where } \theta \in(-1,1),
\end{gathered}
$$

and other $M-|\mathfrak{u}|$ nodes are chosen so that the formula have the highest degree of precision; here, $|\mathfrak{u}|$ is the cardinality, i. e., the number of points of the set $\mathfrak{u}$. It is known (see, for example, $[15$, Ch. 7 , Sect. 1]) that the degree of precision of such formula is $n=2 M-1-|\mathfrak{u}|$. Formulas (2.2) take the form

$$
\int_{-1}^{1} v(t) p(t) d t=\sum_{k=1}^{M} \lambda_{k} p\left(t_{k}\right), \quad p \in \mathscr{P}_{2 M-1-|\mathfrak{u}|}
$$

in what follows, we sometimes will use more accurate (in comparison with (2.2)) notation for nodes $\left\{t_{k}=t_{k}^{\mathfrak{u}}=t_{k}(\mathfrak{u}, v, M)\right\}_{k=1}^{M}$ and weights (coefficients) $\left\{\lambda_{k}=\lambda_{k}^{\mathfrak{u}}=\lambda_{k}(\mathfrak{u}, v, M)\right\}_{k=1}^{M}$ in formula (2.7).

In the case of the empty set $\mathfrak{u}$ (there are no fixed nodes), formula (2.7) is the classical Gauss quadrature formula (see [15, Ch. 7, Sect. 1])). In the cases $\mathfrak{u}=\{-1\}$ and $\mathfrak{u}=\{1\}$, formula (2.7) 
is the left and right Radau quadrature formula, respectively; in the case $\mathfrak{u}=\{-1,1\},(2.7)$ is the Lobatto quadrature formula. It is known (see the references in $[1,3,5]$ ) that formula $(2.7)$ is positive in all these cases.

For each of the sets $\mathfrak{u}$ of fixed nodes from (2.6), the set $\Theta_{M}^{\mathfrak{u}}$ of values of the parameter $\theta \in(-1,1)$ for which quadrature formula (2.7) has positive weights is described in [3, 5]. Such formulas are called quasi Gauss, quasi (left and right) Radau, and quasi Lobatto positive quadrature formulas. In what follows, we consider formula (2.7) with fixed nodes (2.6) only for $\theta \in \Theta_{M}^{\mathfrak{u}}$. Thus, a quadrature formula of the form (2.7) with fixed nodes (2.5) and with fixed nodes (2.6) is positive. The degree of precision of formula (2.7) with fixed nodes (2.5) and with fixed nodes (2.6) is $N=2 M-1-|\mathfrak{u}|$.

The best approximation from below

$$
E_{n, v}^{-}\left(\mathbf{1}_{(a, 1]}\right)=\min \left\{\left\|\mathbf{1}_{(a, 1]}-p_{n}\right\|_{L^{v}(-1,1)}: p_{n} \in \mathscr{P}_{n}^{-}\left(\mathbf{1}_{(a, 1]}\right)\right\}
$$

and an extremal polynomial $p_{n}^{a}=p_{n, a}^{v}$ at which the minimum in (2.8) is attained were found for all values $a \in(-1,1)$ and $n \geq 1$ in the case of the unit weight $v \equiv 1$ in [5] and in the case of an arbitrary weight $v$ in [1]. Results of several statements from [1, Sect. 3] containing the solution of problem (2.8) in the form convenient for us are gathered in the following theorem.

Theorem B [1, Sect. 3]. For $M \in \mathbb{N}, M \geq 3$, the following statements hold.

(1) If the number $a \in(-1,1)$ coincides with one of the nodes of an $M$-point positive quadrature formula (2.7) different from the maximum node, i. e., $a=t_{\nu}^{\mathfrak{u}}, 1 \leq \nu \leq M-1$, then

$$
E_{n, v}^{-}\left(\mathbf{1}_{(a, 1]}\right)=\int_{(a, 1]} v(t) d t-\sum_{k=\nu+1}^{M} \lambda_{k}^{\mathfrak{u}}
$$

for $n=2 M-2-|\mathfrak{u}|$ and $n=2 M-1-|\mathfrak{u}|$ in the case of fixed nodes (2.5) and for $n=2 M-1-|\mathfrak{u}|$ in the case of fixed nodes (2.6). Moreover, the corresponding quadrature formula is extremal, and the polynomial of the best approximation from below is the polynomial $p_{n}^{a} \in \mathscr{P}_{n}^{-}\left(\mathbf{1}_{(a, 1]}\right)$ of degree $n=2 M-2-|\mathfrak{u}|$ for $\mathfrak{u}$ from (2.5) and of degree $n=2 M-1-|\mathfrak{u}|$ for $\mathfrak{u}$ from (2.6) that interpolates the function $\mathbf{1}_{(a, 1]}$ at the nodes of the quadrature formula.

(2) If the maximum node $t_{M}^{\mathfrak{u}}$ of formula (2.7) is less than 1 , then

$$
E_{n, v}^{-}\left(\mathbf{1}_{(a, 1]}\right)=\int_{(a, 1]} v(t) d t
$$

for $t_{M}^{\mathfrak{u}} \leq a<1$ for all $0 \leq n \leq 2 M-1-|\mathfrak{u}|$, and $p^{*} \equiv 0$ is the polynomial of the best approximation from below.

Remark. A statement similar to Theorem B is also valid for the problem $E_{n, v}^{-}\left(\mathbf{1}_{[-1, b)}\right)$ of the best approximation from below to the characteristic function $\mathbf{1}_{[-1, b)}$ of an interval $[-1, b)$. In what follows, we denote by $q_{n}^{b}$ the extremal polynomial in this problem.

Theorem $\mathrm{B}$ and its analog for an interval $[-1, b)$ make it possible to obtain a solution of the problem

$$
E_{n, v}^{-}\left(\mathbf{1}_{(a, b)}\right)=\inf \left\{\left\|\mathbf{1}_{(a, b)}-p_{n}\right\|_{L^{v}(-1,1)}: p_{n} \in \mathscr{P}_{n}^{-}\left(\mathbf{1}_{(a, b)}\right)\right\}
$$

for intervals $(a, b)$ whose end-points are nodes of quadrature formula (2.7). The following statement was obtained in the authors' paper [11].

Theorem $\mathbf{C}$ [11, Theorem 2]. If numbers $a$ and $b,-1<a<b<1$, are nodes of an $M$-point positive quadrature formula (2.7), more exactly,

$$
a=t_{k(a)}^{\mathfrak{u}}, \quad b=t_{k(b)}^{\mathfrak{u}}, \quad k(a)<k(b),
$$


then

$$
E_{n, v}^{-}\left(\mathbf{1}_{(a, b)}\right)=\int_{a}^{b} v(t) d t-\sum_{k(a)<k<k(b)} \lambda_{k}^{\mathfrak{u}}
$$

for $n=2 M-2-|\mathfrak{u}|$ and $n=2 M-1-|\mathfrak{u}|$ in the case of fixed nodes (2.5) and for $n=2 M-1-|\mathfrak{u}|$ in the case of fixed nodes (2.6). Moreover, the corresponding quadrature formula is extremal, and the polynomial of the best approximation from below is the polynomial $\varrho_{n}^{a b}=p_{n}^{a}+q_{n}^{b}-1$ of degree $n=2 M-2-|\mathfrak{u}|$ in the case of fixed nodes (2.5) and of degree $n=2 M-1-|\mathfrak{u}|$ in the case of fixed nodes (2.6).

\section{One-sided approximation to the characteristic function of a spherical cap and a spherical layer}

Theorem 2 and the results of $[1,11]$ presented in Section 2 give a solution of problem (0.6) of the best approximation from below in the space $L\left(\mathbb{S}^{m-1}\right)$ to the characteristic function $\mathbf{1}_{\mathbb{G}(J)}$ of spherical layer $(0.2)$ by the set $\mathscr{P}_{n, m}$ of algebraic polynomials of degree $n$ in $m$ variables for $m \geq 3$ in the following cases.

(1) Approximation from below in $L\left(\mathbb{S}^{m-1}\right)$ to the characteristic function $\mathbf{1}_{\mathbb{C}(a)}$ of a spherical cap $\mathbb{C}(a)=\mathbb{G}(a, 1]$ for $a \in(-1,1)$ which is a node of quadrature formula $(2.7)$. A solution is provided by Theorem 2 and the one-dimensional results from [1] given in Theorem B for the ultraspherical weight

$$
v(t)=\phi(t)=\left(1-t^{2}\right)^{\alpha}, \quad \alpha=(m-3) / 2
$$

(2) Approximation from below in $L\left(\mathbb{S}^{m-1}\right)$ to the characteristic function $\mathbf{1}_{\mathbb{G}(a, b)}$ of a spherical layer $\mathbb{G}(a, b)$, where $-1<a<b<1$ are nodes of positive quadrature formula (2.7). To obtain a solution of this problem, we apply the results of [11] described in Theorem $\mathrm{C}$ for the ultraspherical weight (3.1) and Theorem 2.

\section{Conclusion}

Theorem 2 gives a solution of problem (0.6) in all cases when a solution of the corresponding onedimensional problem is known. For example, Theorem 5 from [11] for the weight $v$ chosen from the condition $v\left(t^{2}\right)|t|=\phi(t), t \in(-1,1)$, i. e., for the Jacobi weight $v(t)=t^{-1 / 2}(1-t)^{(m-3) / 2}, t \in(0,1)$, together with Theorem 2 give the best approximation from below in $L\left(\mathbb{S}^{m-1}\right)$ to the characteristic function $\mathbf{1}_{\mathbb{G}(-h, h)}$ of a spherical layer $\mathbb{G}(-h, h)$ symmetric with respect to "equator" $x_{m}=0$ of the sphere for $h \in(0,1)$.

The results of Section 1, including Theorem 2, can be naturally transferred to the problem of one-sided approximation in the space $L\left(\mathbb{S}^{m-1}\right)$ from above. These results and the corresponding one-dimensional results on the approximation from above to the characteristic functions of intervals from $[1,11]$, make it possible to obtain a write solution of the problem $e_{n, m}^{+}\left(\mathbf{1}_{\mathbb{G}(J)}\right)$ on the best approximation from above in the space $L\left(\mathbb{S}^{m-1}\right)$ to the characteristic function $\mathbf{1}_{\mathbb{G}(J)}$ of spherical layer $(0.2)$ by the set $\mathscr{P}_{n, m}$ of algebraic polynomials of degree $n$ in $m$ variables, at least, in the two cases described in Section 3.

\section{Acknowledgements}

The authors are grateful to Professor V.V. Arestov for the attention to their study and useful discussion of the results. 


\section{REFERENCES}

1. Babenko A. G., Deikalova M. V., Revesz Sz. G. Weighted one-sided integral approximations to characteristic functions of intervals by polynomials on a closed interval. Proc. Steklov Inst. Math., 2017. Vol. 297, Suppl. 1. P. S11-S18. DOI: 10.1134/S0081543817050029

2. Babenko A. G., Kryakin Yu. V., Yudin V.A., One-sided approximation in $L$ of the characteristic function of an interval by trigonometric polynomials. Proc. Steklov Inst. Math., 2013. Vol. 280, Suppl. 1. P. S39S52. DOI: 10.1134/S0081543813020041

3. Beckermann B., Bustamante J., Martinez-Cruz R., Quesada J. M. Gaussian, Lobatto and Radau positive quadrature rules with a prescribed abscissa. Calcolo, 2014. Vol. 51, No. 2. P. 319-328. DOI: $10.1007 / \mathrm{s} 10092-013-0087-3$

4. Bojanic R., DeVore R. On polynomials of best one-sided approximation. Enseign. Math., 1966. Vol. 12. P. 139-164.

5. Bustamante J., Martínez-Cruz R., Quesada J.M. Quasi orthogonal Jacobi polynomials and best one-sided $L_{1}$ approximation to step functions. J. Approx. Theory, 2015. Vol. 198. P. 10-23. DOI: $10.1016 /$ j.jat.2015.05.001

6. Bustamante J., Quesada J.M., Martínez-Cruz R. Best one-sided $L_{1}$ approximation to the Heaviside and sign functions. J. Approx. Theory, 2012. Vol. 164, No. 6. P. 791-802. DOI: 10.1016/j.jat.2012.02.006

7. Dai F., Xu Y. Approximation Theory and Harmonic Analysis on Spheres and Balls. New York: Springer Science \& Business Media, 2013. 440 p. DOI: 10.1007/978-1-4614-6660-4

8. Deikalova M. V. The Taikov functional in the space of algebraic polynomials on the multidimensional Euclidean sphere. Math Notes, 2008. Vol. 84, No. 3-4. P. 498-514. DOI: 10.1134/S0001434608090228

9. Deikalova M.V. Integral approximation of the characteristic function of a spherical cap by algebraic polynomials. Proc. Steklov Inst. Math., 2011. Vol. 273, Suppl. 1. P. S74-S85. DOI: 10.1134/S0081543811050087

10. Deikalova M. V. Several extremal approximation problems for the characteristic function of a spherical layer. Proc. Steklov Inst. Math., 2012. Vol. 277, Suppl. 1. P. S79-S92. DOI: 10.1134/S0081543812050094

11. Deikalova M. V., Torgashova A. Yu. One-sided integral approximation of the characteristic function of an interval by algebraic polynomials. Trudy Inst. Mat. $i$ Mekh. UrO RAN [Proc. of Krasovskii Institute of Mathematics and Mechanics of the UB RAS], 2018. Vol. 24, No. 4. P. 110-125. (In Russian) DOI: 10.21538/0134-4889-2018-24-4-110-125

12. Dunford N., Schwartz J. T. Linear Operators. Part I: General Theory. New York: Wiley-Interscience, 1988. 872 p.

13. Gorbachev D. V. Izbrannye zadachi teorii funkcij $i$ teorii priblizhenij $i$ ih prilozheniya [Selected Problems in Functional Analysis and Approximation Theory and Their Applications]. Tula: TulGU, 2004. 152 p. (In Russian)

14. Korneichuk N. P., Ligun A. A., Doronin V. G. Approksimaciya s ogranicheniyami [Approximation with Constraints]. Kiev: Naukova Dumka, 1982. 254 p. (In Russian)

15. Krylov V. I. Approximate Calculation of Integrals. Mineola, New York: Dover Publ. 2006. 368 p.

16. Li X.-J., Vaaler J.D. Some trigonometric extremal functions and the Erdös-Turán type inequalities. Indiana Univ. Math. J., 1999. Vol. 48, No. 1. P. 183-236. DOI: 10.1512/iumj.1999.48.1508

17. Motornyi V.P., Motornaya O. V., Nitiema P. K. One-sided approximation of a step by algebraic polynomials in the mean. Ukrainian Math. J., 2010. Vol. 62, No. 3. P. 467-482. DOI: 10.1007/s11253-010-0366-y 